\title{
RESPONSE OF SUNFLOWER (Helianthus annuus L.) TO PHOSPHORUS AND NITROGEN FERTILIZATION UNDER RAINFED CONDITIONS, BLUE NILE STATE-SUDAN
}

Salih, M.N.T.

Department of Soil Sciences, Faculty of Agriculture,

University of Upper Nile, El-Renk, Sudan

Received: November 26, 2012

Accepted: June 10, 2013

\section{SUMMARY}

Two field experiments were conducted on a typic chromusterts, fine, smectitic, isohyperthermic soil series in the Damazin Research Station Farm during two seasons (2005/06 and 2006/07). The objective was to investigate the effect of phosphorus (P) and nitrogen $(\mathrm{N})$ and their interactions on sunflower (Helianthus annuus L.) growth, seed and oil yield. A randomized complete block design with four replicates was used to execute the experiments. Plant height, dry matter and seed yield were significantly increased by $\mathrm{N}$ and $\mathrm{P}$ applications in both seasons. The average yield of sunflower seed in the two seasons was 0.486 tons $\cdot \mathrm{ha}^{-1}$ for the control and 4.893 tons $^{\cdot} \mathrm{ha}^{-1}$ for the highest fertilizer treatment $\left(\mathrm{N}_{80} \mathrm{P}_{80}\right)$. Oil seed \% gave significant increases in the two seasons due to $\mathrm{P}, \mathrm{N}$ treatments and their interaction, and the average oil seed was $31.3 \%$ for the control compared to $37.5 \%$ for the highest treatment $\left(\mathrm{N}_{80} \mathrm{P}_{80}\right)$. Phosphorus alone had pronounced effect on oil seed \% in the two seasons. There were mutually synergistic effects between $\mathrm{N}$ and $\mathrm{P}$ which significantly $(\mathrm{P}<0.01)$ promoted growth and yield of sunflower. Phosphorus enhanced nitrogen uptake and nitrogen use efficiency and vice versa. At all $\mathrm{N}$ applications, phosphorus use efficiency tended to decrease with increase in the rate of $\mathrm{P}$ application. The highly significant response of sunflower to $\mathrm{P}$ and $\mathrm{N}$ fertilizers warrant recommending their application under the rainfed conditions of Damazin, Sudan.

Key words: sunflower (Helianthus annuus L.), phosphorus, nitrogen, semiarid conditions, rainfed conditions, vertisols

\section{INTRODUCTION}

The majority of cultivated lands of the Sudan lie within the rainfed sector; some of them are semi-mechanized and mono-cropped and the rest belong to the traditional subsistence sector. Despite the short periods of rainy season, in most parts rainfed agriculture remains the cheapest economic activity, but it depends on rain- 
fall. Fertility loss is the main common problem associated with shifting cultivation without fertilization practiced on such soils. The deterioration of soil fertility on these soils may be attributed to natural and human activity such as erosion factors, beside mono-cropping, poor agricultural management and practice and inadequate rain fall.

Soil fertility and, hence, sunflower yields in semi arid regions of the Sudan have been declining, because of lack of fertilization and lack of crop rotation. Production of sunflower in Sudan started 1990-1991 and the cultivated area reached 293000 feddans ( 1 feddan $=4200 \mathrm{~m}^{2}$ ) mainly in the rainfed area with average yield of $99 \mathrm{~kg}$ / feddan (Faisal et al., 2005). Sunflower research program implemented by Agricultural Research Corporation of the Sudan reported that commercial production of sunflower started in 1986 in the rainfed sector. Areas under the crop had declined dramatically in recent years, productivity is low and production was as low as 0.2 ton per feddan in 1998/1999 according to Faisal et al. (2005). Gorashi and Elzein, (2005) reported the average yield in the Blue Nile State as about 0.2 tons/ feddan, and the cultivated area is about 18000 feddan in season 1999/2000.

Application of $\mathrm{P}$ affects sunflower growth and yield. The results by Muralidharudu et al. (2003) showed high increase in the sunflower plant height, head diameter, shoot dry matter yield and seed yield, as a response to applied phosporus. The response to applied $\mathrm{P}$ was more pronounced on soils low in available phosphorus than in the other soils. Rodriguez et al. (1998) observed that under $P$ deficiencies sunflower showed a reduction in the rate of leaf expansion and in photosynthetic rate per unit of leaf area.

Fixation of applied phosphorus fertilizer is expected to be high under these conditions. However, the summer rainfall in the southern part of northern Sudan exceeds $700 \mathrm{~mm}$, which is sufficient for many food and cash crops, and the soil $\mathrm{pH}$ is near neutral (Table 2) which may decrease P fixation. Research results by Abdalla and El Mahi (1986) indicated good positive response to P and P N interactions of rainfed sunflower grown in the vertisols of the Blue Nile State, Sudan.

Thus, the objective of this study was to investigate the effect of $\mathrm{N}$ and $\mathrm{P}$ fertilizers and their interactions on rainfed sunflower grown in the neutral to slightly acid soils of the Blue Nile State, Sudan.

\section{MATERIALS AND METHODS}

Two experiments were carried out at two sites in the Agricultural Research Station Farm, Damazin, Sudan, located at longitude $34^{\circ} 22^{\prime} \mathrm{E}$, latitude $11^{\circ} 47^{\prime} \mathrm{N}$ and altitude $470 \mathrm{~m}$. Soil is classified as typic chromusterts, fine, smectitic, isohyperthermic (Soil Survey staff, 1976). Sunflower (Helianthus annuus L.) was cultivated in the first site in the growing season of 2005/06 and in the other neighboring site in the season of 2006/07. Surface soil samples $(0-30 \mathrm{~cm})$ were collected and analyzed. Physical and chemical properties of these soils are presented in Table 1. Soil nitro- 
gen was determined by a modified semi-micro Kjeldhal method (Hesse, 1971). Available soil phosphorus was extracted by sodium bicarbonate and determined spectrometrically, using stannous-chloride - molybdenum-blue method described by Olsen and Sommers (1982). Other soil properties were determined according to the methods of soil analysis described by Page et al. (1982).

Table 1: Some chemical and physical properties of Damazin soils cultivated in seasons 2005/ 06 and 2006/07

\begin{tabular}{|c|c|c|c|c|c|c|c|c|c|c|c|c|c|c|}
\hline \multicolumn{15}{|c|}{ Chemical properties } \\
\hline \multirow{3}{*}{ Season } & \multirow{3}{*}{$\begin{array}{c}\mathrm{pH} \\
\text { paste }\end{array}$} & \multirow{2}{*}{$\mathrm{EC}$} & \multirow{2}{*}{$\mathrm{CaCO}_{3}$} & \multirow{2}{*}{ Nitrogen } & \multirow{2}{*}{$\begin{array}{l}\text { Organic } \\
\text { carbon }\end{array}$} & Soluk & ble ca & tions & Solut & e anions & Exchan & cations & \multirow{3}{*}{ CEC } & \multirow{2}{*}{ Available $\mathrm{P}$} \\
\hline & & & & & & $\mathrm{Na}$ & $\mathrm{Ca}$ & $\mathrm{Mg}$ & $\mathrm{Cl}$ & $\mathrm{HCO}_{3}$ & $\mathrm{Na}$ & $\mathrm{K}$ & & \\
\hline & & $\mathrm{dSm}^{-1}$ & & $\%$ & & & & $\mathrm{~m}$ & & & $\mathrm{Cmol}($ & $\mathrm{kg}^{-1}$ soil & & $\mathrm{mg} \cdot \mathrm{kg}^{-1} \mathrm{soi}$ \\
\hline $2005 / 06$ & 7.00 & 0.23 & 0.60 & 0.049 & 0.156 & 0.68 & 2.5 & 0.5 & 0.5 & 0.5 & 1.10 & 0.65 & 54 & 5.0 \\
\hline $2006 / 07$ & 6.92 & 0.35 & 0.00 & 0.031 & 0.782 & 1.20 & 2.3 & 0.8 & 2.0 & 1.5 & 0.24 & 0.63 & 53 & 5.4 \\
\hline
\end{tabular}

\begin{tabular}{|c|c|c|c|c|c|c|c|c|c|c|c|}
\hline \multicolumn{12}{|c|}{ Physical properties } \\
\hline \multirow{3}{*}{ Season } & \multicolumn{4}{|c|}{ Mechanical analysis } & \multicolumn{2}{|l|}{ Saturation } & \multicolumn{3}{|c|}{ Soil moisture } & \multirow{2}{*}{\multicolumn{2}{|c|}{$\begin{array}{c}\text { Bulk density } \\
\mathrm{g} \cdot \mathrm{cm}^{-3}\end{array}$}} \\
\hline & \multirow{2}{*}{ CS } & \multirow{2}{*}{ FS } & \multirow{2}{*}{$\mathrm{Si}$} & \multirow{2}{*}{ C } & \multirow{2}{*}{$\%$} & \multirow{2}{*}{$0.3 \mathrm{bar}$} & \multirow{2}{*}{15 bar } & \multirow{2}{*}{ AWC } & \multirow{2}{*}{ Vol \% } & & \\
\hline & & & & & & & & & & Dry & Moist \\
\hline $2005 / 06$ & 7 & 3 & 20 & 70 & 78 & 42.5 & 22.7 & 22.8 & 33.7 & 1.77 & 1.19 \\
\hline 2006/07 & 3 & 3 & 26 & 68 & 60 & 47.7 & 24.9 & 23.3 & 34.5 & 1.80 & 1.16 \\
\hline
\end{tabular}

Two factorial experiments were conducted using randomized complete block design with four replicates in both seasons. The levels of fertilizer used were 0,20 , 40 and $80 \mathrm{~kg} \cdot \mathrm{ha}^{-1}$ for both $\mathrm{P}$ and N. Phosphorus was applied as triple-superphosphate (before ploughing and planting) and $\mathrm{N}$ as urea (split dose after sowing and one month later), and the fertilizer combinations were assigned as 16 different treatments. The plot area was $60 \mathrm{~m}^{2}(12 \times 5 \mathrm{~m})$, ridged $75 \mathrm{~cm}$ apart. The sowing date in the first season was 3-4 July and the harvest date was 12-14 November, while in second season the dates were 16-17 July and 5-7 December for sowing and harvest, respectively. Plant height was recorded 109 and 107 days after sowing in the first and second seasons, respectively. Dry matter and seed yield were recorded after harvest. Oil content in seed (in \%) was determined at Faculty of Agriculture laboratories, University of Khartoum, Shambat, Sudan, using hexane solvent in soxhlet extraction apparatus, according to the procedure described by American Oil Chemists Society (1995). Whole plant samples were randomly taken after harvest for determination of $\mathrm{P}$ and $\mathrm{N}$ contents, using nitro-vanado-molybdate method for determination of phosphorus (Cottoni, 1980) and modified Kjeldhal method, for determination of nitrogen (Chapman and Pratt, 1961). Nitrogen use efficiency and phosphorus use efficiency were calculated as seed yield per unit area ( $\left.\mathrm{kg} \mathrm{seed} \mathrm{ha}^{-1}\right)$ produced per unit of $\mathrm{N}$ and $\mathrm{P}$ applied $\left(\mathrm{kg} \mathrm{N} \mathrm{ha}^{-1}\right.$ and $\left.\mathrm{kg} \mathrm{P} \mathrm{ha}^{-1}\right)$.

The collected data were statistically analyzed using Statistical Analysis Software SAS version 9.1 (SAS 2009). 


\section{RESULTS AND DISCUSSION}

Nitrogen and phosphorus fertilization of rainfed cultivation is generally not practiced in dry land farming of the clay plains of northern Sudan. This is probably because water deficit remains the major limiting factor of crop production, and that nitrogen fertilization in particular may encourage vegetative growth, increasing evapotranspiration and, thus, exacerbates the water deficit problem.

Rainfed as well as irrigated crops in the Sudan central clay plain have shown erratic responses to $\mathrm{P}$ fertilization. This is in spite of the fact that the $\mathrm{NaHCO}_{3}$ extractable $\mathrm{P}$ rarely exceeded $2-3 \mathrm{mg} \cdot \mathrm{kg}^{-1}$ in most of these alkaline calcareous dry lands (Dawelbeit et al., 2007). Such values are considered deficient according to the American Society of Agronomy standards (Olsen and Sommers, 1982). Rainfall of the southern part of northern Sudan, however, usually ranges between 600 and $700 \mathrm{~mm}$ (Table 2) during a four month growing summer season, and the soil $\mathrm{pH}$ therein is near neutral which decreases $\mathrm{P}$ fixation.

Table 2: Accumulation of rainfall in millimetres $(\mathrm{mm})$, per day (d) during growing seasons 2005/06 (a) and 2006/2007 (b)

\begin{tabular}{|c|c|c|c|c|c|c|c|c|c|c|c|c|}
\hline \multicolumn{13}{|l|}{ a) } \\
\hline \multicolumn{2}{|c|}{ May } & \multicolumn{2}{|c|}{ June } & \multicolumn{2}{|c|}{ July } & \multicolumn{2}{|c|}{ August } & \multicolumn{2}{|c|}{ September } & \multicolumn{2}{|c|}{ October } & \multirow{2}{*}{ Total season } \\
\hline d & $\mathrm{mm}$ & $\mathrm{d}$ & $\mathrm{mm}$ & d & $\mathrm{mm}$ & $\mathrm{d}$ & $\mathrm{mm}$ & $\mathrm{d}$ & $\mathrm{mm}$ & $\mathrm{d}$ & $\mathrm{mm}$ & \\
\hline 4 & 20.0 & 4 & 15.0 & 3 & 42.0 & 3 & 4.0 & 1 & 3.0 & 10 & 4.0 & \\
\hline 23 & 3.0 & 6 & 3.5 & 6 & 13.0 & 4 & 39.0 & 5 & 3.0 & 19 & 3.0 & \\
\hline 25 & 27 & 9 & 23.5 & 13 & 21.0 & 6 & 22.0 & 9 & 21.5 & 20 & 1.5 & \\
\hline 26 & 14.5 & 10 & 40.0 & 17 & 37.0 & 10 & 63.0 & 14 & 4.0 & & & \\
\hline \multirow[t]{7}{*}{27} & 7.5 & 13 & 3.0 & 21 & 36.0 & 14 & 8.0 & 18 & 5.0 & & & \\
\hline & & 19 & 6.0 & 25 & 64.0 & 15 & 38.0 & 21 & 16.0 & & & \\
\hline & & 20 & 3.0 & 28 & 8.0 & 16 & 43.0 & 26 & 37.0 & & & \\
\hline & & 23 & 40.0 & & & 17 & 16.0 & 28 & 1.5 & & & \\
\hline & & 28 & 4.5 & & & 18 & 10.0 & & & & & \\
\hline & & & & & & 27 & 4.0 & & & & & \\
\hline & & & & & & 31 & 1.0 & & & & & \\
\hline \multicolumn{13}{|c|}{ Total } \\
\hline \multicolumn{2}{|c|}{70.0} & \multicolumn{2}{|c|}{138.5} & \multicolumn{2}{|c|}{221.0} & 248.0 & & \multicolumn{2}{|c|}{91.0} & \multicolumn{2}{|c|}{8.5} & 777.0 \\
\hline
\end{tabular}

b)

\begin{tabular}{|c|c|c|c|c|c|c|c|c|c|c|c|c|}
\hline \multicolumn{2}{|c|}{ May } & \multicolumn{2}{|c|}{ June } & \multicolumn{2}{|c|}{ July } & \multicolumn{2}{|c|}{ August } & \multicolumn{2}{|c|}{ September } & \multicolumn{2}{|c|}{ October } & \multirow{2}{*}{ Total season } \\
\hline$d$ & $\mathrm{~mm}$ & $d$ & $\mathrm{~mm}$ & $\mathrm{~d}$ & $\mathrm{~mm}$ & $d$ & $\mathrm{~mm}$ & $\mathrm{~d}$ & $\mathrm{~mm}$ & $\mathrm{~d}$ & $\mathrm{~mm}$ & \\
\hline & & 12 & 10.0 & 16 & 8.0 & 1 & 5.5 & 1 & 6.0 & 17 & 33.0 & \\
\hline & & 13 & 7.0 & 18 & 13.0 & 4 & 43.0 & 8 & 8.0 & 22 & 42.0 & \\
\hline & & 18 & 16.0 & 20 & 47.0 & 14 & 59.0 & 10 & 17.0 & 29 & 2.0 & \\
\hline & & 20 & 18.0 & 22 & 8.0 & 23 & 26.0 & 14 & 2.0 & & & \\
\hline & & 22 & 11.5 & 25 & 43.0 & 25 & 58.0 & 17 & 2.0 & & & \\
\hline & & 25 & 13.0 & 28 & 26.5 & 29 & 2.0 & 23 & 25.0 & & & \\
\hline & & & & & & & & 26 & 5.0 & & & \\
\hline \multicolumn{13}{|c|}{ Total } \\
\hline & & & & & & & & & 5.0 & & 7.0 & 556.5 \\
\hline
\end{tabular}


Table 1 shows low total $\mathrm{N}$, and that available $\mathrm{P}\left(\mathrm{NaHCO}_{3}\right.$ extractable- $\left.\mathrm{P}\right)$ was 5 $\mathrm{mg} \cdot \mathrm{kg}^{-1}$ soil in the soil of the experimental sites. Olsen and Sommers (1982) consider that crops grown in such soils are likely to respond to $\mathrm{P}$ fertilization. In the two seasons there was significant $(\mathrm{P}<0.01)$ increase in plant height $(109$ days after sowing) due to $\mathrm{P}$ application, also nitrogen application gave significant increase in plant height in both seasons (Tables 3 and 4). The results of the present experiment also indicated a significant $(\mathrm{P}<0.01)$ interactive effect of $\mathrm{P}$ and $\mathrm{N}$ application on sunflower plant height in both seasons (Table 3 and 4).

Table 3: Effect of N, P and their interaction on plant height, dry matter production seed yield and nutrients uptake of sunflower grown in season 2005/2006

\begin{tabular}{|c|c|c|c|c|c|c|}
\hline Treatments & $\begin{array}{c}\text { Plant height } \\
\mathrm{cm}\end{array}$ & $\begin{array}{c}\text { Dry matter } \\
\mathrm{kg} \cdot \mathrm{ha}^{-1}\end{array}$ & $\begin{array}{c}\text { Seed yield } \\
\text { ton }^{-h^{-1}}\end{array}$ & $\begin{array}{c}\mathrm{N} \text { uptake } \\
\mathrm{kg} \cdot \mathrm{ha}^{-1}\end{array}$ & $\begin{array}{c}\text { P uptake } \\
\mathrm{kg}^{\prime} \mathrm{ha}^{-1}\end{array}$ & $\begin{array}{l}\text { Oil } \\
\%\end{array}$ \\
\hline \multicolumn{7}{|l|}{$P\left(k g \cdot h^{-1}\right)$} \\
\hline 0 & $92.49 \mathrm{a}$ & $2170 \mathrm{a}$ & $0.898 a$ & $64.82 \mathrm{a}$ & $5.42 \mathrm{a}$ & $23.18 \mathrm{a}$ \\
\hline 20 & $101.45 b$ & $3325 \mathrm{~b}$ & $2.180 \mathrm{~b}$ & $82.81 \mathrm{~b}$ & $10.48 b$ & $25.01 \mathrm{~b}$ \\
\hline 40 & $113.46 \mathrm{c}$ & $3599 \mathrm{c}$ & $3.144 \mathrm{c}$ & $97.81 \mathrm{c}$ & $13.17 \mathrm{c}$ & $28.44 \mathrm{c}$ \\
\hline 80 & $141.59 \mathrm{~d}$ & $3906 \mathrm{~d}$ & $3.884 \mathrm{~d}$ & $118.70 \mathrm{~d}$ & $16.04 \mathrm{~d}$ & $32.00 \mathrm{~d}$ \\
\hline \multicolumn{7}{|l|}{$\mathrm{N}\left(\mathrm{kg} \cdot \mathrm{ha}^{-1}\right)$} \\
\hline 0 & $95.20 \mathrm{a}$ & $2724 a$ & $1.520 \mathrm{a}$ & $61.20 \mathrm{a}$ & $8.73 \mathrm{a}$ & $24.21 \mathrm{a}$ \\
\hline 20 & $108.0 \mathrm{~b}$ & $3262 \mathrm{~b}$ & $2.410 \mathrm{~b}$ & $84.70 \mathrm{~b}$ & $11.11 \mathrm{~b}$ & $26.89 a b$ \\
\hline 40 & $118.6 \mathrm{c}$ & $3446 \mathrm{c}$ & $3.010 \mathrm{c}$ & $99.70 \mathrm{c}$ & $12.16 \mathrm{c}$ & $27.87 \mathrm{~b}$ \\
\hline 80 & $127.2 \mathrm{~d}$ & $3570 \mathrm{c}$ & $3.170 \mathrm{c}$ & $118.50 \mathrm{~d}$ & $13.12 \mathrm{~d}$ & $29.66 \mathrm{c}$ \\
\hline \multicolumn{7}{|c|}{$\mathrm{N}^{\star} \mathrm{P}$ combination $\left(\mathrm{kg} \cdot \mathrm{ha}^{-1}\right)$} \\
\hline $\mathrm{P}_{00} \mathrm{~N}_{00}$ & $82.98 \mathrm{a}$ & $1501 a$ & $0.335 a$ & $33.03 a$ & $3.00 a$ & $21.99 a$ \\
\hline $\mathrm{P}_{00} \mathrm{~N}_{20}$ & $90.63 \mathrm{a}$ & $2182 a$ & $0.886 a$ & $62.53 a$ & $4.99 a$ & $22.35 \mathrm{a}$ \\
\hline $\mathrm{P}_{00} \mathrm{~N}_{40}$ & $96.38 \mathrm{a}$ & $2400 a$ & $1.177 \mathrm{a}$ & $79.40 a$ & $6.56 \mathrm{a}$ & $23.34 a b$ \\
\hline $\mathrm{P}_{00} \mathrm{~N}_{80}$ & 99.98 a & $2597 a$ & $1.195 \mathrm{a}$ & $84.33 a$ & $7.15 a$ & $25.08 \mathrm{~b}$ \\
\hline $\mathrm{P}_{20} \mathrm{~N}_{00}$ & $88.70 \mathrm{a}$ & $2934 b$ & $1.574 \mathrm{C}$ & $61.48 \mathrm{~b}$ & $8.55 b$ & $21.99 a$ \\
\hline $\mathrm{P}_{20} \mathrm{~N}_{20}$ & $96.03 \mathrm{a}$ & $3198 b$ & $1.785 b$ & $70.35 b$ & $10.12 b$ & 25.49 bc \\
\hline $\mathrm{P}_{20} \mathrm{~N}_{40}$ & $107.73 b$ & $3549 b$ & $2.562 \mathrm{~b}$ & $88.10 \mathrm{~b}$ & $11.04 b$ & $25.97 \mathrm{bcc}$ \\
\hline $\mathrm{P}_{20} \mathrm{~N}_{80}$ & $113.38 b$ & $3622 b$ & $2.799 \mathrm{~b}$ & $111.3 b$ & $12.19 b$ & $26.58 \mathrm{bcc}$ \\
\hline $\mathrm{P}_{40} \mathrm{~N}_{00}$ & $95.30 \mathrm{~b}$ & $2987 b$ & $1.468 \mathrm{~b}$ & $67.73 b c$ & $9.89 c$ & $26.39 \mathrm{bcc}$ \\
\hline $\mathrm{P}_{40} \mathrm{~N}_{20}$ & $102.05 \mathrm{~b}$ & $3661 c$ & $2.842 \mathrm{c}$ & $87.30 \mathrm{c}$ & $13.13 \mathrm{c}$ & $27.58 \mathrm{de}$ \\
\hline $\mathrm{P}_{40} \mathrm{~N}_{40}$ & $121.50 \mathrm{c}$ & $3851 c$ & $4.074 \mathrm{c}$ & $103.55 b$ & $14.50 \mathrm{c}$ & $28.66 \mathrm{e}$ \\
\hline $\mathrm{P}_{40} \mathrm{~N}_{80}$ & $134.98 \mathrm{c}$ & $3896 c$ & $4.193 c$ & $132.60 \mathrm{c}$ & $15.16 \mathrm{c}$ & $31.15 f$ \\
\hline $\mathrm{P}_{80} \mathrm{~N}_{00}$ & $114.03 \mathrm{c}$ & $3473 c$ & $2.685 d$ & $82.75 b$ & $13.47 d$ & $26.49 \mathrm{bcc}$ \\
\hline $\mathrm{P}_{80} \mathrm{~N}_{20}$ & $143.23 \mathrm{c}$ & $4005 d$ & $4.138 \mathrm{~d}$ & $118.65 d$ & $16.19 d$ & $32.16 \mathrm{f}$ \\
\hline $\mathrm{P}_{80} \mathrm{~N}_{40}$ & $148.60 \mathrm{~d}$ & $3983 c$ & $4.226 \mathrm{~d}$ & $127.65 c$ & $16.56 d$ & $33.50 \mathrm{fg}$ \\
\hline $\mathrm{P}_{80} \mathrm{~N}_{80}$ & $160.50 \mathrm{~d}$ & $4163 d$ & $4.486 \mathrm{~d}$ & $145.6 d$ & $17.97 \mathrm{~d}$ & $35.84 \mathrm{~h}$ \\
\hline CV\% & 9.24 & 7.147 & 20.63 & 17.17 & 9.67 & 5.97 \\
\hline
\end{tabular}

Means in the same columns followed by the same letter(s) are not significantly different at 0.05 probability level according to Duncan's multiple range test. 
The synergistic effect of phosphorus and nitrogen on each other was also clear on dry matter production and seed yield (Figure 1) in the two seasons. The increases in dry matter and yield due to $\mathrm{N}$ fertilizer application were expected as these vertisols are usually deficient in nitrogen (Table 1). This deficiency is generally attributed to the low amount of soil organic matter as mentioned by Syers et al. (2001). Tables 3 and 4 show that sunflower seed yield was highly significantly $(\mathrm{P}<0.01)$ increased due to $\mathrm{P}$ and $\mathrm{N}$ application in the two seasons. The increase in yield also due to high $\mathrm{P}$ applications on vertisols could be attributed to the fixing capacity of these soils even at neutral pH as stated by Wild (1988). N and P uptake by sunflower had rather similar trends to those in seed yield and plant height (Tables 3 and 4). In both seasons nitrogen and phosphorus uptake showed highly significant increases $(\mathrm{P}<0.01)$ due to $\mathrm{P}$ and $\mathrm{N}$, and their interactions as compared with the control.

Table 4: Effect of N, P and their interaction on plant height, dry matter production seed yield and nutrients uptake of sunflower grown in season 2006/2007

\begin{tabular}{|c|c|c|c|c|c|c|}
\hline \multirow{2}{*}{ Treatments } & Plant height & Dry matter & Seed yield & $\mathrm{N}$ uptake & P uptake & Oil \\
\hline & $(\mathrm{cm})$ & $\mathrm{kg} \cdot \mathrm{ha}^{-1}$ & ton $\cdot \mathrm{ha}^{-1}$ & $\mathrm{~kg} \cdot \mathrm{ha}^{-1}$ & $\mathrm{~kg} \cdot \mathrm{ha}^{-1}$ & $\%$ \\
\hline \multicolumn{7}{|l|}{$P\left(\mathrm{~kg} \cdot \mathrm{ha}^{-1}\right)$} \\
\hline 0 & $96.4 \mathrm{a}$ & $2351 a$ & $1.616 a$ & $77.5 \mathrm{a}$ & $07.51 \mathrm{a}$ & $26.07 \mathrm{a}$ \\
\hline 20 & $137.0 \mathrm{~b}$ & 2946 b & $3.211 \mathrm{~b}$ & $94.1 \mathrm{ab}$ & $12.29 \mathrm{bc}$ & $31.36 \mathrm{~b}$ \\
\hline 40 & $160.7 \mathrm{c}$ & 3404 bc & $4.298 \mathrm{c}$ & $112.9 \mathrm{bc}$ & 11.07 b & $33.01 \mathrm{~b}$ \\
\hline 80 & $174.7 \mathrm{~d}$ & $3558 \mathrm{c}$ & $4.806 \mathrm{~d}$ & $132.9 \mathrm{c}$ & $14.47 \mathrm{c}$ & $35.63 \mathrm{c}$ \\
\hline \multicolumn{7}{|l|}{$\mathrm{N}\left(\mathrm{kg} \cdot \mathrm{ha}^{-1}\right)$} \\
\hline 0 & $132.6 \mathrm{a}$ & $2626 a$ & $2.580 \mathrm{a}$ & $74.6 \mathrm{a}$ & $8.50 \mathrm{a}$ & $26.55 \mathrm{a}$ \\
\hline 20 & $139.3 \mathrm{~b}$ & $2818 \mathrm{a}$ & $3.430 \mathrm{~b}$ & $92.1 \mathrm{a}$ & $9.95 \mathrm{a}$ & $28.51 \mathrm{a}$ \\
\hline 40 & $143.2 \mathrm{~b}$ & 3499 b & $3.500 \mathrm{c}$ & $123.0 \mathrm{~b}$ & $13.36 \mathrm{~b}$ & $35.13 \mathrm{~b}$ \\
\hline 80 & $153.6 \mathrm{c}$ & $3318 b$ & $4.050 \mathrm{~d}$ & $125.6 \mathrm{~b}$ & $13.54 \mathrm{~b}$ & $35.88 \mathrm{~b}$ \\
\hline \multicolumn{7}{|c|}{$\mathrm{N}^{\star} \mathrm{P}$ combination $\left(\mathrm{kg} \cdot \mathrm{ha}^{-1}\right)$} \\
\hline $\mathrm{P}_{00} \mathrm{~N}_{00}$ & $84.6 \mathrm{a}$ & $1467 \mathrm{a}$ & $0.636 a$ & $33.9 a$ & $04.03 \mathrm{a}$ & $24.56 \mathrm{a}$ \\
\hline $\mathrm{P}_{00} \mathrm{~N}_{20}$ & $93.2 \mathrm{a}$ & $2009 a$ & $0.840 \mathrm{a}$ & $58.5 a$ & 06.27 a & $26.20 \mathrm{abc}$ \\
\hline $\mathrm{P}_{00} \mathrm{~N}_{40}$ & $96.1 \mathrm{a}$ & 3007 a & $1.449 \mathrm{a}$ & $112.1 \mathrm{a}$ & $10.42 \mathrm{a}$ & $27.66 \mathrm{bc}$ \\
\hline $\mathrm{P}_{00} \mathrm{~N}_{80}$ & $111.8 \mathrm{a}$ & $2923 a$ & $1.737 \mathrm{a}$ & $105.6 \mathrm{~b}$ & $09.33 \mathrm{a}$ & $25.87 a b$ \\
\hline $\mathrm{P}_{20} \mathrm{~N}_{00}$ & $124.8 \mathrm{~b}$ & 3227 c & $2.410 \mathrm{~b}$ & $94.9 \mathrm{c}$ & $10.09 \mathrm{c}$ & $28.67 \mathrm{~cd}$ \\
\hline $\mathrm{P}_{20} \mathrm{~N}_{20}$ & $129.6 \mathrm{~b}$ & $3477 \mathrm{~d}$ & $3.535 \mathrm{~b}$ & 113.7 c & $11.48 \mathrm{c}$ & $26.88 \mathrm{abc}$ \\
\hline $\mathrm{P}_{20} \mathrm{~N}_{40}$ & $140.1 \mathrm{~b}$ & $3433 \mathrm{c}$ & $2.854 \mathrm{~b}$ & $114.5 \mathrm{a}$ & $13.01 \mathrm{~b}$ & $33.47 \mathrm{e}$ \\
\hline $\mathrm{P}_{20} \mathrm{~N}_{80}$ & $153.3 \mathrm{~b}$ & $3478 \mathrm{~b}$ & $4.045 \mathrm{~b}$ & 128.2 c & $14.59 \mathrm{c}$ & $36.43 \mathrm{f}$ \\
\hline $\mathrm{P}_{40} \mathrm{~N}_{00}$ & $152.9 \mathrm{c}$ & $3512 d$ & $3.708 \mathrm{c}$ & $99.5 \mathrm{c}$ & $12.10 \mathrm{~d}$ & $24.99 a$ \\
\hline $\mathrm{P}_{40} \mathrm{~N}_{20}$ & $160.4 \mathrm{c}$ & $2591 \mathrm{~b}$ & $4.258 \mathrm{c}$ & $80.3 \mathrm{~b}$ & $09.30 \mathrm{~b}$ & $30.43 d$ \\
\hline $\mathrm{P}_{40} \mathrm{~N}_{40}$ & $163.2 \mathrm{c}$ & $3146 \mathrm{~b}$ & $4.460 \mathrm{c}$ & 106.9 a & $12.39 \mathrm{c}$ & $37.77 \mathrm{f}$ \\
\hline $\mathrm{P}_{40} \mathrm{~N}_{80}$ & $166.1 \mathrm{c}$ & $3534 b$ & $4.767 \mathrm{c}$ & $89.8 \mathrm{a}$ & $10.47 b$ & $38.87 \mathrm{fg}$ \\
\hline $\mathrm{P}_{80} \mathrm{~N}_{00}$ & $168.1 \mathrm{~d}$ & 2295 b & $3.550 \mathrm{~d}$ & $70.2 \mathrm{~b}$ & 07.77 b & $27.98 \mathrm{bcd}$ \\
\hline $\mathrm{P}_{80} \mathrm{~N}_{20}$ & $174.1 \mathrm{~d}$ & $3193 \mathrm{c}$ & $5.084 \mathrm{~d}$ & $116.0 \mathrm{c}$ & $12.76 \mathrm{~d}$ & $30.53 \mathrm{~d}$ \\
\hline $\mathrm{P}_{80} \mathrm{~N}_{40}$ & $173.4 \mathrm{~d}$ & $4410 \mathrm{~d}$ & $5.230 \mathrm{~d}$ & $168.9 \mathrm{~b}$ & $18.32 \mathrm{~d}$ & $41.67 \mathrm{~g}$ \\
\hline $\mathrm{P}_{80} \mathrm{~N}_{80}$ & $183.0 \mathrm{~d}$ & $4334 \mathrm{c}$ & $5.300 \mathrm{~d}$ & $176.3 \mathrm{~d}$ & $19.04 \mathrm{~d}$ & $42.33 \mathrm{~g}$ \\
\hline CV\% & 5.5 & 19.10 & 20.78 & 23.65 & 23.6 & 8.22 \\
\hline
\end{tabular}

Means $f$ in the same columns ollowed by the same letter(s) are not significantly different at 0.05 probability level according to Duncan's multiple range test. 

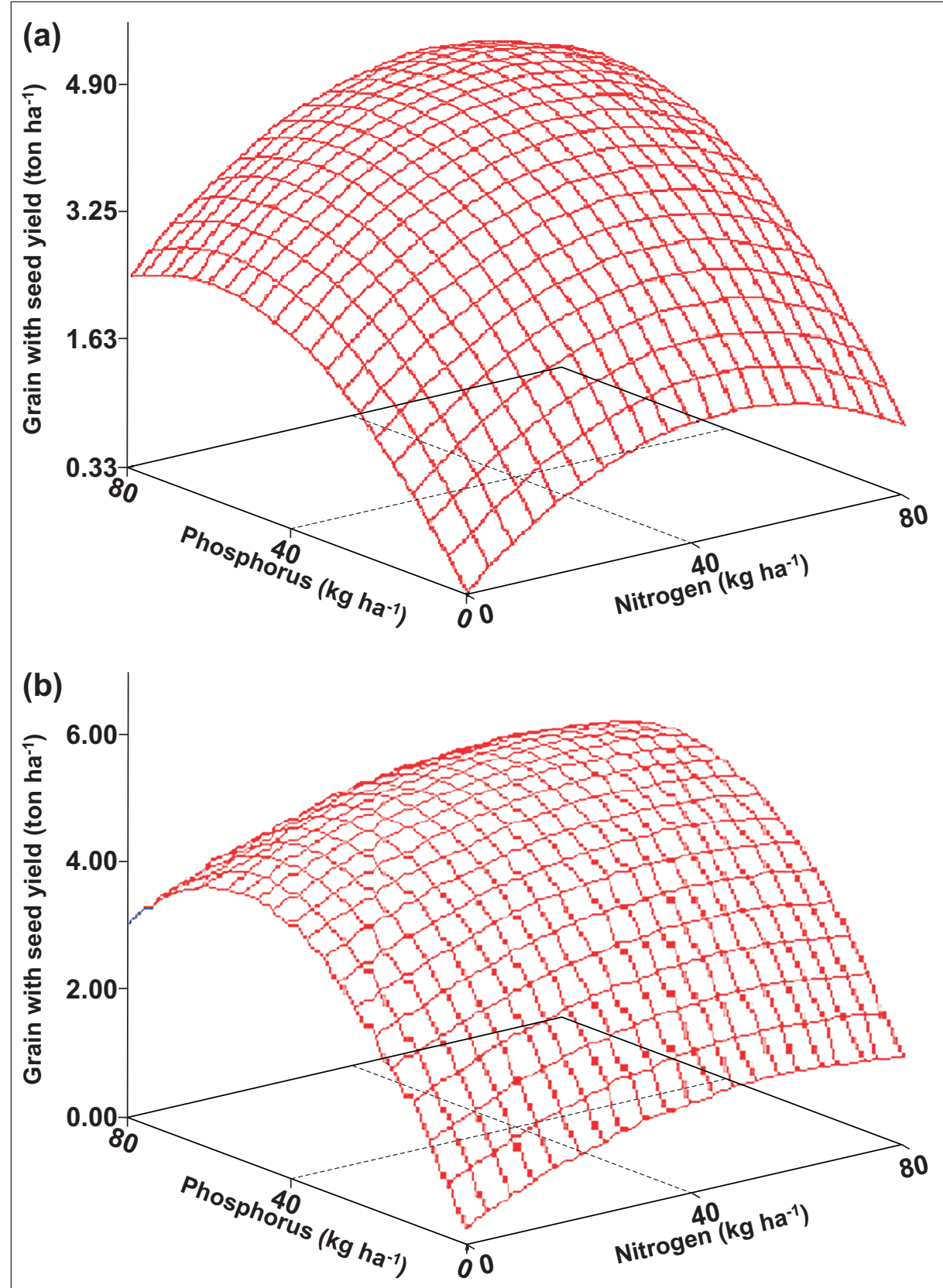

Figure 1. Response surface curve showing the interactive effect of added phosphorus and nitrogen on sunflower seed yield in season 2005/06 (a) and season 2006/07 (b).

(a) Seed yield $=0.11+0.26 P+0.18 N-0.002 P^{2}+0.0005 N P-0.002 N^{2},\left(R^{2}=0.85\right)$.

(b) Seed yield $=1.97+0.41 P+0.12 N-0.003 P^{2}+0.001 N P-0.001 N^{2},\left(R^{2}=0.84\right)$. 
Phosphorus gave significant increase in sunflower oil content in both seasons, while nitrogen gave significant increase only in the first season. P application seems to increase oil yield per unit area through the increase in seed yield without a reduction in \% oil due to dilution with increase in yield. This study conclude that $\mathrm{P}$ is recommended to increase oil percentage, Furthermore, nitrogen seems to play a vital role in increasing the quality of sunflower oil and protein content by increasing seed weight and yield

Nitrogen use efficiency (NUE) decreased with increasing $\mathrm{N}$ rate nearly at every $\mathrm{P}$ application level, in the two seasons (Table 5). At specific N level, despite the effect of P, NUE was not regular but it seems to be rather additive. This may be attributed to increase in vegetative growth (especially in the second season) and, consequently, higher evapotranspiration resulting in negative soil moisture $\backslash$ high nitrogen rate of application interaction. Application of $40 \mathrm{~kg} \mathrm{P}$ in the first season and $80 \mathrm{~kg} \mathrm{P}$ in the second season per ha tended to give the highest NUE. P application, possibly increased root growth according to Rajendran and Veeraputhiran (2001), leading to increase in both water and nitrogen absorption from the soil. In the two seasons PUE approximately increased with increasing $\mathrm{N}$ application rate at every $\mathrm{P}$ level, and PUE seems to be decreased with increasing $\mathrm{P}$ rate of application. At zero $\mathrm{P}$ application, NUE decreased with increasing $\mathrm{N}$ application in the first season and increased with increasing $\mathrm{N}$ application up to $\mathrm{N}_{40}$ level then decreased at $\mathrm{N}_{80}$ in the second season. These results suggest that at southern Blue Nile environment sunflower may respond to high $\mathrm{P}$ additions for better NUE and respond to lower addition of $\mathrm{P}$ for the best PUE. However, these results concerning nutrients use efficiency on seed yield bases rather than the oil content to get the real efficiency.

Table 5: Effect of $\mathrm{P}$ and $\mathrm{N}$ application on nutrients use efficiency of sunflower

\begin{tabular}{lcccc}
\hline \multirow{2}{*}{$\mathrm{NP}$} & \multicolumn{2}{c}{ season 2005/06 } & \multicolumn{2}{c}{ season 2006/07 } \\
\cline { 2 - 5 } & NUE $^{\star}$ & $\mathrm{PUE}^{\star}$ & 0.00 & $\mathrm{PUE}^{\star}$ \\
\hline $\mathrm{P}_{00} \mathrm{~N}_{00}$ & 0.00 & 0.00 & 0.00 \\
$\mathrm{P}_{00} \mathrm{~N}_{20}$ & 27.5 & 0.00 & 10.20 & 0.00 \\
$\mathrm{P}_{00} \mathrm{~N}_{40}$ & 21.05 & 0.00 & 20.33 & 0.00 \\
$\mathrm{P}_{00} \mathrm{~N}_{80}$ & 10.75 & 0.00 & 13.76 & 0.00 \\
$\mathrm{P}_{20} \mathrm{~N}_{00}$ & 0.00 & 61.95 & 0.00 & 88.70 \\
$\mathrm{P}_{20} \mathrm{~N}_{20}$ & 10.55 & 44.95 & 56.25 & 33.69 \\
$\mathrm{P}_{20} \mathrm{~N}_{40}$ & 24.70 & 69.25 & 55.45 & 70.25 \\
$\mathrm{P}_{20} \mathrm{~N}_{80}$ & 15.31 & 80.20 & 42.61 & 81.75 \\
$\mathrm{P}_{40} \mathrm{~N}_{00}$ & 0.00 & 28.33 & 0.00 & 76.80 \\
$\mathrm{P}_{40} \mathrm{~N}_{20}$ & 68.70 & 48.90 & 27.50 & 85.45 \\
$\mathrm{P}_{40} \mathrm{~N}_{40}$ & 65.15 & 72.43 & 18.8 & 75.27 \\
$\mathrm{P}_{40} \mathrm{~N}_{80}$ & 34.06 & 74.95 & 75.27 & 75.75 \\
$\mathrm{P}_{80} \mathrm{~N}_{00}$ & 0.00 & 29.38 & 0.00 & 36.43 \\
$\mathrm{P}_{80} \mathrm{~N}_{20}$ & 72.65 & 40.65 & 76.70 & 53.05 \\
$\mathrm{P}_{80} \mathrm{~N}_{40}$ & 38.53 & 38.11 & 42.00 & 45.44 \\
$\mathrm{P}_{80} \mathrm{~N}_{80}$ & 22.51 & 41.14 & 21.88 & 44.54 \\
\hline
\end{tabular}

$\mathrm{NUE}^{\star}=\mathrm{kg}$ seed $/ \mathrm{kg} \mathrm{N}$. PUE* $=\mathrm{kg}$ seed $/ \mathrm{kg}$ P. 
Since P uptake by sunflower did not exceed $40 \mathrm{~kg} \mathrm{ha}^{-1}$ per season and that $\mathrm{N}$ uptake was well above the highest $\mathrm{N}\left(80 \mathrm{~kg} \mathrm{~N} \mathrm{ha}^{-1}\right)$ application, it seems that for soil and crop yield conservation $\mathrm{N}_{80} \mathrm{P}_{40}(80 \mathrm{~kg} \mathrm{~N}+40 \mathrm{~kg}$ P) may be the most appropriate for sunflower crop for the range and fertilizer addition used in these experiments. Seed yield read with NUE and PUE showing in Table 5, may indicate that the treatment $\mathrm{N}_{80} \mathrm{P}_{80}$ should be recommended if the cash return from increase in seed yield (above $300 \mathrm{~kg} \mathrm{ha}^{-1}$ ) price did not exceeded that of the price of excess fertilizer added $\left(40 \mathrm{~kg} \mathrm{ha}^{-1}\right)$ in the last treatment. Also $\mathrm{N}_{80} \mathrm{P}_{80}$ treatment gave significantly higher $\mathrm{N}$ (protein for animals) and oil content in the first season over and above highest seed yield. In order to maintain $\mathrm{N}$ status in these soils heavy $\mathrm{N}$ fertilization should be practiced, and the incorporation of crop residues may be necessary to obtain sustainable soil fertility and high yields of sunflower crop studied in these experiments.

Interaction effect of $\mathrm{N}$ and $\mathrm{P}$ on sunflower seed yield can be represented by the response surface curve (Figures $1 \mathrm{a}$ and $\mathrm{b}$ ) and the relation was expressed by regression equations land 2 in the first and second seasons respectively.

Seed yield $=0.32+0.16 \mathrm{P}+0.018 \mathrm{~N}-0.002 \mathrm{P}^{2}+0.0005 \mathrm{NP}-0.002 \mathrm{~N}^{2}$ ton $\cdot \mathrm{ha}^{-1}$,

$$
\left(\mathrm{R}^{2}=0.85\right)
$$

Seed yield $=0.619+0.41 \mathrm{P}+0.12 \mathrm{~N}-0.003 \mathrm{P}^{2}+0.001 \mathrm{NP}-0.001 \mathrm{~N}^{2}$ ton $\cdot \mathrm{ha}^{-1}$,

$$
\left(\mathrm{R}^{2}=0.842\right)
$$

Equation (2) shows several folds contribution of $\mathrm{P}$ as compared to N. The highest sunflower seed yield (5.3 tons $\cdot$ ha $^{-1}$ ) was given by $\mathrm{N}_{80} \mathrm{P}_{80}$ treatment.

\section{CONCLUSIONS}

This study suggests that intensive $\mathrm{P}$ and $\mathrm{N}$ fertilization should be practiced in the neutral to slightly acidic Vertisols of South Eastern Sudan. Heavy N fertilization under adequate $\mathrm{P}$ supply is necessary to obtain sustainable soil fertility and high crop yields. The amounts of $\mathrm{N}$ taken by plants exceed any economically practical $\mathrm{N}$ fertilizer to be added. Thus, other remedial measures like incorporating crop residues in the soil may be necessary to obtain sustainable soil conditions and crop yield under high $\mathrm{P}$ fertilization. The calculations of NUE proved that 72.6 and 76.7 $\mathrm{kg}$ seed/kg N are the best NUE at the two seasons, respectively, obtained at $\left(\mathrm{N}_{80} \mathrm{P}_{20}\right)$, while calculation of PUE indicated $80.2 \mathrm{~kg} \mathrm{seed} / \mathrm{kg} \mathrm{P}$ at the first and $88.7 \mathrm{~kg}$ seeds/ $\mathrm{kg} \mathrm{P}$ at the second season, respectively, at $\mathrm{N}_{20} \mathrm{P}_{00}$ and $\mathrm{N}_{20} \mathrm{P}_{80}$ level. The highest yield, however, was obtained at the highest $\mathrm{N}$ and $\mathrm{P}$ applications 


\section{REFERENCES}

Abdalla, A.K. and El Mahi, Y.E., 1986. Effect of N, P and P N interactions on some field crops of some soils in the Blue Nile State, Sudan. U. of K. J. Agric. Sci.13(2): 60-77.

American Oil Chemists Society (AOCS), 1995. Official and Recommended Methods of American Oil Chemists Society. $4^{\text {th }}$ edition (edited by D. Firestone). Champaign, IL, AOCS Press.

Chapmann, H.D. and Pratt, P. F., 1961. Method of analysis for soils, plant, and water. Univ, of California, Division of Agric. Sci. Berkeley, Calif., U.S.A. Pp. 44-45.

Cottonie, A., 1980. Methods of plant analysis. In: FAO (ed.). Soil and Plant Testing as a bases of fertilizer recommendations. FAO Soils Bull. 38/2. FAO of the UN Rome. Pp. 94-100.

Dawelbeit, S.E., Salih, F.M., Dahab, O.A. and Ahmid, S.E.H., 2007. Current Research in Fertilizer use in Irrigated Agriculture in Sudan. $13^{\text {th }}$ AFA Int'l Annual Fertilizers Forum and Exhibition 6-8 February 2007. Sharm El-Sheikh, Egypt.

Faisal, M. Ali, Mohamed ELNaseeh M. Osman and ELNaiem A. Ali, 2005. Sunflower: A proposed new crop for the Gash Delta. Proceedings of the Meetings of the National Crop Husbandry Committee $37^{\text {th }}$, pp. $77-83$.

Gorashi, M. and Elzein, A., 2005. Annual Report (2005-06). Department of Planning and Information, General management of Agricultural, Ministry of Agricultural, Animal Resources and Irrigation, Blue Nile State, Sudan.

Hesse, P.R., 1971. A text book of soil chemical analysis. John Murray, Ltd., 50 Albemarle st., London, Wix, 4BD, UK.

Muralidharudu, Y., Murthy, I.Y.LN., Reddy, K.P.C., Reddy, B.N. and Chandranath, H.T., 2003. Response of Sunflower (Helianthus annuus L.) to Phosphorus Application in Vertisols. Helia 26(39): 47-154.

OECD (Organization for Economic Co-operation and Development), 2006. Safety Assessment of Transgenic Organisms: OECD Consensus Document, Volumes I and II, OECD Publishing.

Olsen, S.R., Cole, C.V., Watanabe, F. S. and Dean, L.A.,1954. Estimation of available phosphorus in soils by extraction with sodium bicarbonate. U. S. Dep. of Agric. Circ. 939.

Olsen, S.R., and Sommers, L.E.. 1982. Phosphorus. In: A.L. Page (ed.) Methods of Soil Analysis. Part 2. $2^{\text {nd }}$ ed. Agronomy 9: 403-430.

Page, A.L., Miller, R.H. and Keeney, D.R., 1982. Methods of Soil Analysis. Part 2. Chemicaland MicrobialProperties. Amer. Soc. Agron. No. 9 (Part 2) in the Agronomy series. ASA, SSSA, Madison. Pp. 1159.

Rajendran, K. and Veeraputhiran, R. 2001. Phosphorus nutrients in sunflower - a review. Agricultural Reviews 22(1): 68-70.

Rodriguez, D., Zubillaga, M.M., Ploschuck, E., Keltjens, W., Goudriaan, J. and Lavado, R., 1998. Leaf area expansion and assimilate prediction in sunflower growing under low phosphorus conditions. Plant Soil 202, 133-47.

SAS Institute Inc. 2009. Version 9.1, Cary, NC, U. S. A.

Soil Survey Staff 1976. Simi-detailed soil survey report, SSA No. 78, Soil Survey Administration, Wad Medani, Sudan.

Syers, J.K.. Frits, W.T., de Vries, P. and Nyamudeza, P., 2001. Part 2. Country Papers and National perspectives on the Management of Vertisols.

Vong, N.Q. and Murata, Y., 1977. Studies on the Physiological Characteristics of $\mathrm{C}_{3}$ and $\mathrm{C}_{4}$ Crop Species. 1- The effects of air temperature on the apparent photosynthesis, dark respiration, and nutrient absorption of some crops. Japan Journal of Crop Science 46( 1): 45-52.

Wild, A., 1988. Plant nutrients in soils: Phosphorus. In: Wild, A. (ed.). Russell's: Soil conditions and Plant Growth. pp. 689-694. 